\title{
Correction: Exosomal miR-25-3p from mesenchymal stem cells alleviates myocardial infarction by targeting pro-apoptotic proteins and $\mathrm{EZH} 2$
}

\author{
Yi Peng, Ji-Ling Zhao, Zhi-Yong Peng, Wei-Fang Xu and Guo-Long Yu (i)
}

Correction to: Cell Death \& Disease

https://doi.org/10.1038/s41419-020-2545-6

published online 5 May 2020

Since online publication of this article, the authors noticed that an incorrect image was used in Fig. 2a. The corrected Fig. 2 has been provided below.

In addition, the last sentence of the "Material and methods" section describing the Detection of exosomes uptake by cardiomyocytes contained incorrect information on labelling. The full and corrected paragraph is provided below.

Isolated exosomes were incubated with $3.3 \mu \mathrm{L}$ of Alexa FlourTM 488 C5 Maleimide $(200 \mu \mathrm{g} / \mathrm{mL}$, A10254, Thermo Scientific, San Jose, CA, USA) for $1 \mathrm{~h}$ at room temperature. The labelling was disturbed by passing through the exosome spin column (MW3000, 4484449,
Thermo Scientific, San Jose, CA, USA), according to manufacturer's instruction. The labelled exosomes were washed out and resuspended with $1 \mathrm{~mL}$ of serum free OptiMEM (31985088, Thermo Scientific, San Jose, CA, USA). For each well in a 4-well plate, $250 \mu \mathrm{L}$ labelled exosomes were incubated with primary cardiomyocytes in the standard cell culture condition for $4 \mathrm{~h}$ at $37^{\circ} \mathrm{C}$. Cardiomyocytes were then counterstained with CellTracker Deep Red dye and mounted with ProLong Gold antifade mountants without DAPI (\#P36934, Thermo Scientific, San Jose, CA, USA). The cells labelling with Alexa 488 (green) under the microscope were considered as positive cells containing exosomes.

The authors apologise for these errors.

Published online: 12 October 2020 


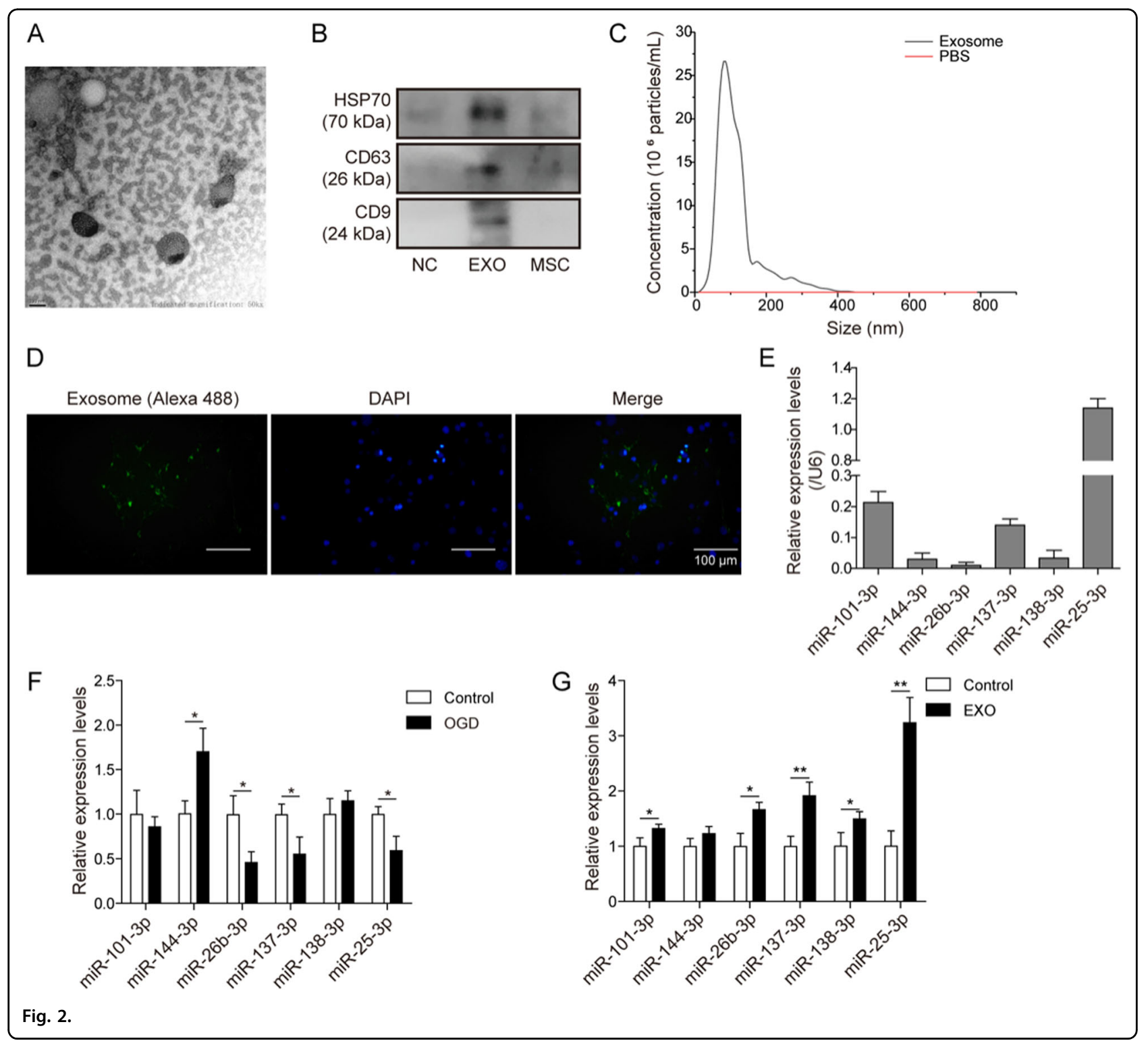

